\title{
Numerical Analysis of a Waveguide-Based Demultiplexer With Two Multiple-Layer Filters
}

\author{
Junji Yamauchi, Member, IEEE, Masaru Koshihara, and Hisamatsu Nakano, Fellow, IEEE
}

\begin{abstract}
A demultiplexer whose input waveguide intersects perpendicularly to one of the output waveguides is proposed and investigated by the finite-difference beam-propagation method. A $1.55-\mu \mathrm{m}$-pass $/ 1.31-\mu \mathrm{m}$-reflection property is achieved regardless of polarizations. Detailed study of power evaluation is also made on the basis of the Poynting vector.
\end{abstract}

Index Terms-Beam-propagation method (BPM), multiple-layer filter, optical waveguide, polarization dependence.

\section{INTRODUCTION}

C ONSIDERABLE effort has been made to develop a low crosstalk wavelength-division-multiplexing bidirectional transceiver. A multiple-layer filter has been used as a demultiplexing element [1]-[5] because of its compactness and simplicity. It should be noted that a multiple-layer filter has strong polarization dependence when the light incident angle to the filter is large. Consequently, we usually design the transceiver so that the incident angle to the filter may be small, leading to the fact that the input waveguide is nearly parallel to the output waveguide. This arrangement, however, results in a long device length.

In this study, we adopt another arrangement, in which the input waveguide is perpendicular to one of the output waveguides, maintaining small polarization dependence. For this, we employ two multiple-layer filters, in which the incident angle to each filter remains to be $22.5^{\circ}$. Note that this type of configuration can be efficiently analyzed by the finite-difference beam-propagation method (BPM), when the multiple-layer interface is placed parallel to the propagating beam direction [6]. We should, however, pay attention to the method for evaluating power. Before investigating the demultiplexing property of the filter, we reveal that the power can be calculated correctly by including the effect of the first derivative of the field with respect to the propagating beam direction. Simulation based on the BPM demonstrates that a $1.55-\mu \mathrm{m}$-pass $/ 1.31-\mu \mathrm{m}$-reflection property is achieved regardless of polarizations.

\section{Evaluation OF POWER IN THE BPM ANALYsis}

In this section, we clarify the relation between the exact power defined by the Poynting vector and the approximate one defined by the squared norm under the slowly varying envelope approximation (SVEA).

Manuscript received July 20, 2004; revised September 8, 2004

The authors are with Faculty of Engineering, Hosei University, Koganei, Tokyo 184-8584, Japan (e-mail: j.yma@k.hosei.ac.jp).

Digital Object Identifier 10.1109/LPT.2004.839376
We assume a planar geometry and start from Maxwell's equations

$$
\begin{aligned}
\nabla \times \mathcal{E} & =-j \omega \mu_{0} \mathcal{H} \\
\nabla \times \mathcal{H} & =j \omega \epsilon_{0} n^{2} \mathcal{E}
\end{aligned}
$$

where $\epsilon_{0}$ and $\mu_{0}$ are the free-space permittivity and permeability, respectively. $\omega$ is the angular frequency and $n=n(x, z)$ is the refractive index profile of a planar waveguide. We derive the expression for the exact power [7].

For the transverse-electric (TE) wave, the power is expressed as

$$
P_{\mathrm{TE}}=\frac{1}{2} \operatorname{Re} \int-\mathcal{E}_{y} \mathcal{H}_{x}^{*} d x=-\frac{1}{2 \omega \mu_{0}} \operatorname{Re} \int j \mathcal{E}_{y} \frac{\partial \mathcal{E}_{y}^{*}}{\partial z} d x
$$

After expressing the field $\mathcal{E}_{y}(x, z)$ as $\mathcal{E}_{y}(x, z)=$ $E(x, z) \exp \left(-j k_{0} n_{0} z\right)$, where $n_{0}$ is some reference refractive index yet to be chosen, we obtain

$$
P_{\mathrm{TE}}=\frac{1}{2 \omega \mu_{0}} \operatorname{Re} \int\left(k_{0} n_{0}|E|^{2}-j E \frac{\partial E^{*}}{\partial z}\right) d x .
$$

Similarly, for the transverse-magnetic (TM) wave, the power is expressed as

$$
P_{\mathrm{TM}}=\frac{1}{2} \operatorname{Re} \int \mathcal{E}_{x} \mathcal{H}_{y}^{*} d x=\frac{1}{2 \omega \epsilon_{0}} \operatorname{Re} \int j \frac{\mathcal{H}_{y}^{*}}{n^{2}} \frac{\partial \mathcal{H}_{y}}{\partial z} d x .
$$

Again, expressing the field $\mathcal{H}_{y}(x, z)=$ $H(x, z) \exp \left(-j k_{0} n_{0} z\right)$, we obtain

$$
P_{\mathrm{TM}}=\frac{1}{2 \omega \epsilon_{0}} \operatorname{Re} \int\left(k_{0} n_{0} \frac{|H|^{2}}{n^{2}}+j \frac{H^{*}}{n^{2}} \frac{\partial H}{\partial z}\right) d x .
$$

It should be noted that typing errors of [7, eqs. (8.5) and (8.6)] are corrected in (5) and (6).

Within the framework of the SVEA, the $E$-field satisfies the relation $\left|k_{0} n_{0} E\right| \gg|\partial E / \partial z|$. The same is also true for the $H$-field. This means that the second terms in (4) and (6) can be neglected, provided that the SVEA is maintained, and hence, the power is simply expressed as the squared norm. This fact, however, suggests that the second terms in (4) and (6) should be included for the case where the variation of the field cannot be negligible in the propagation direction. In this letter, to obtain the $E$ - and $H$-fields, we employ the $(1,1)$ Padé-operator-based BPM [8] together with the power-conserving algorithm [7] and the fourth-order finite-difference schemes [9], [10].

\section{DISCUSSION}

Before considering a modified demultiplexer, we first discuss the characteristics of the conventional demultiplexer composed of a multiple-layer filter with a vertex angle of $\theta_{1}=45^{\circ}$ shown 


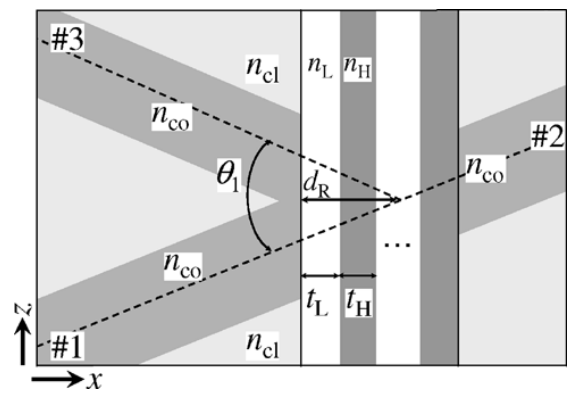

Fig. 1. Configuration of the conventional demultiplexer $\left(n_{\mathrm{co}}=1.536, n_{\mathrm{cl}}=\right.$ $\left.1.532, n_{H}=2.25\left(\mathrm{TiO}_{2}\right), n_{L}=1.45\left(\mathrm{SiO}_{2}\right)\right)$.

in Fig. 1. We excite the field of either TE or TM wave from Waveguide \#1, and intend to extract a $1.55-\mu \mathrm{m}$ wave in Waveguide \#2 and a $1.31-\mu \mathrm{m}$ wave in Waveguide \#3 for both polarizations. The refractive indexes of the core and cladding are taken to be $n_{\mathrm{co}}=1.536$ and $n_{\mathrm{cl}}=1.532$, respectively. The normalized frequency of the waveguide is almost equal to $V=1.5$ at $1.31 \mu \mathrm{m}$. The number of layers is taken to be 20 . The thicknesses of the layers are determined by

$$
t_{H \text { or } L}=\frac{\lambda_{S}}{\left(4 n_{H \text { or } L} \cos \left(\frac{\theta_{1}}{2}\right)\right)}
$$

where $n_{H}$ and $n_{L}$ are the corresponding refractive indexes in the high- and low-index layers, respectively. In this analysis, $\lambda_{S}$ is chosen to be $1.17 \mu \mathrm{m}$. The transverse and longitudinal sampling widths are taken to be $\Delta x=t_{H} / 6$ and $\Delta z=\Delta x / \cot \left(\theta_{1} / 2\right)$, respectively.

The effective reflection distance designated as $d_{R}$ should be chosen to maximize reflectivity. Preliminary calculation shows that the maximum reflectivities for $\theta_{1}=45^{\circ}$ at $1.31 \mu \mathrm{m}$ are obtained at $d_{R}=2\left(t_{H}+t_{L}\right)$ for the TE wave and $d_{R}=4\left(t_{H}+\right.$ $\left.t_{L}\right)$ for the TM wave. It follows that $d_{R}$ is fixed to be $3\left(t_{H}+t_{L}\right)$ to maintain reasonable reflectivities for both waves.

To assess the method for evaluating the power, we calculate the normalized power as a function of propagation distance. The results for both TE and TM waves are shown in Fig. 2, in which the data obtained with and without the second $(j)$ term are shown for comparison. The reference refractive index $n_{0}$ is fixed to be $\left(\beta / k_{0}\right) \sin \left(\theta_{1} / 2\right)$ ( $\beta$ is the propagation constant of the waveguide) or adaptively renewed as the field propagates [11]. It is observed that the power evaluated without the $j$ th term experiences variation, particularly during the propagation of the filter region (this fact is more pronounced as $\theta_{1}$ is decreased). In contrast, the normalized power is found to be stable, when the $j$ th term is included. It should also be noted that the result with fixed $n_{0}$ is almost the same as that with adaptively determined $n_{0}$, provided the $j$ th term is included for power evaluation.

To further validate the use of the BPM, we next evaluate the demultiplexing characteristics and compare the results with those obtained with the finite-difference time-domain method [12]. Fig. 3 shows the reflectivity and transmissivity for the TM wave as a function of wavelength. Good agreement is found to exist between both results. We now adopt the BPM for the following analysis due to its computational efficiency.

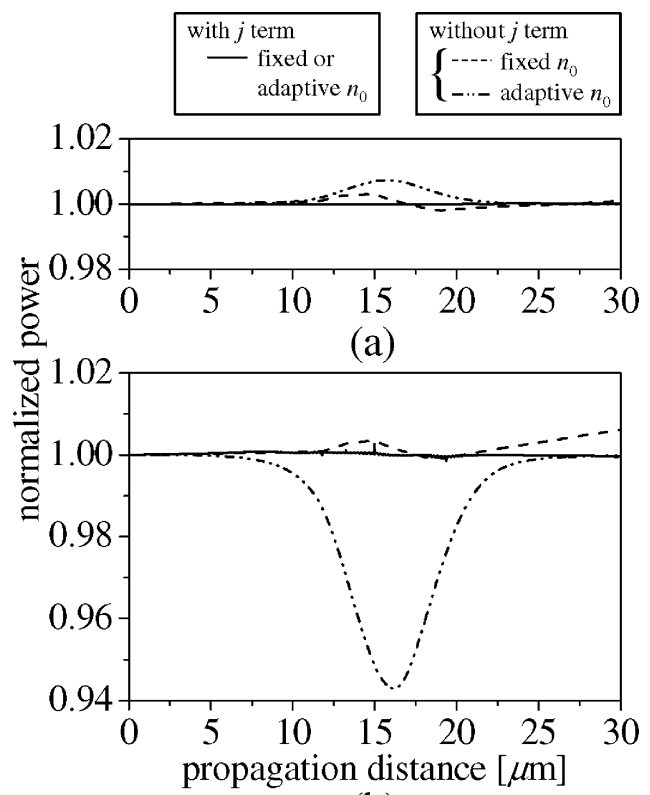

(b)

Fig. 2. Normalized power as a function of propagation distance at $1.55 \mu \mathrm{m}$ $\left(\theta_{1}=45^{\circ}\right)$. (a) TE wave. (b) TM wave.

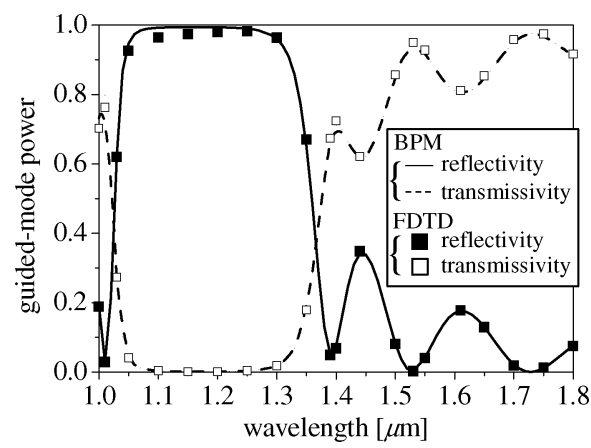

Fig. 3. Wavelength response of the conventional demultiplexer for TM wave $\left(\theta_{1}=45^{\circ}\right)$

We should also note in Fig. 3 that a $1.55-\mu \mathrm{m}$-pass $/ 1.31-\mu \mathrm{m}$ -reflection property is achieved for the TM wave, which contrasts with that obtained with $\theta_{1}=90^{\circ}$. This encourages us to devise a configuration with two multiple-layer filters in such a way that an input waveguide intersects perpendicularly to an output waveguide.

Fig. 4 shows a proposed configuration with two multiplelayer filters. This configuration leads to the advantage that the characteristics of Filters \#1 and \#2 can be chosen arbitrarily, depending on applications. In this letter, Filter $\# 1$ is taken to be identical with Filter \#2. The vertex angle is $\theta_{1}=45^{\circ}$, so that the input Waveguide \#1 intersects perpendicularly to the output Waveguide \#4. In the BPM analysis, we first analyze Filter \#1, and then the output field from Filter \#1 is used as an input field for the analysis of Filter \#2. Note that since the waveguides are weakly guiding, the loss caused at the intersection is small. This means that the overall properties may be approximately evaluated by the square of the result obtained with a single multiple-layer filter.

Wavelength responses of reflectivity and transmissivity of the modified demultiplexer are shown in Fig. 5(a) and 


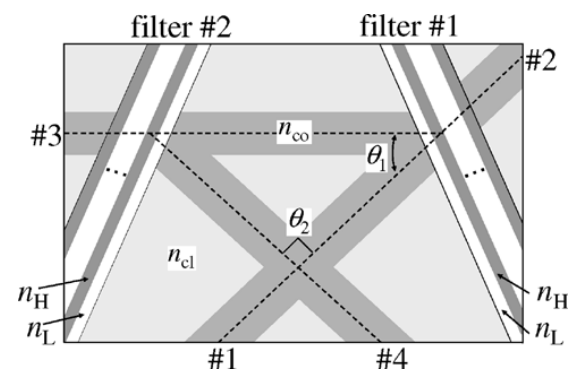

Fig. 4. Configuration of a modified demultiplexer $\left(n_{\mathrm{co}}=1.536, n_{\mathrm{cl}}=\right.$ $\left.1.532, n_{H}=2.25\left(\mathrm{TiO}_{2}\right), n_{L}=1.45\left(\mathrm{SiO}_{2}\right), \theta_{1}=45^{\circ}, \theta_{2}=90^{\circ}\right)$.

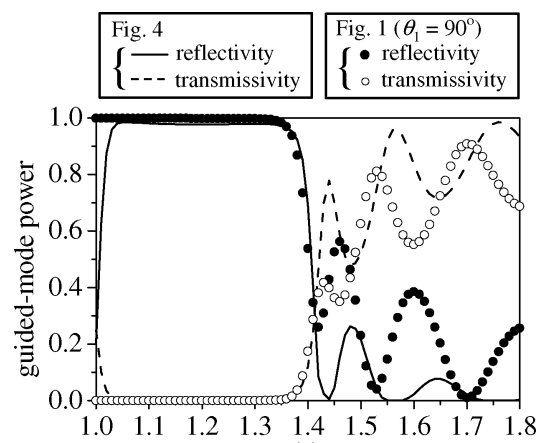

(a)

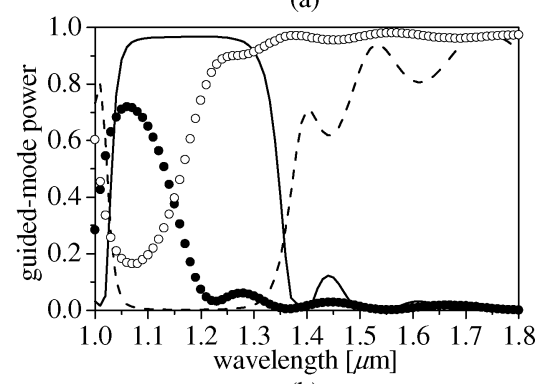

(b)

Fig. 5. Wavelength responses of the modified demultiplexer. (a) TE wave. (b) TM wave. For $\theta_{1}=90^{\circ}$, the effective reflection distance is changed to $d_{R}=t_{H}+t_{L}$, which provides the maximum reflectivity for the TE wave at $1.31 \mu \mathrm{m}$.

(b). It is found that the modified demultiplexer achieves a $1.55-\mu \mathrm{m}$-pass $/ 1.31-\mu \mathrm{m}$-reflection property for both TE and TM waves. Both reflectivity and transmissivity are calculated to be about $90 \%$ regardless of polarizations. In contrast, such characteristics cannot be obtained for the conventional demultiplexer with $\theta_{1}=90^{\circ}$, particularly for the TM wave shown in Fig. 5(b).

To further improve the characteristics, we have also studied the effects of introducing an absentee layer placed at the middle of the multiple layers. As a result, both reflectivity and transmissivity are improved to be more than $94 \%$ regardless of polarizations.

\section{CONCLUSION}

The finite-difference BPM has been used to analyze a multiple-layer-based demultiplexer whose input waveguide intersects perpendicularly to one of the output waveguides. The method for evaluating the power in the BPM is discussed on the basis of the Poynting vector. As a result, the $1.55-\mu \mathrm{m}$-pass $/ 1.31-\mu \mathrm{m}$-reflection property is achieved regardless of polarizations.

\section{REFERENCES}

[1] Y. Inoue, T. Oguchi, Y. Hibino, S. Suzuki, M. Yanagisawa, K. Moriwaki, and Y. Yamada, "Filter-embedded wavelength-division multiplexer for hybrid-integrated transceiver based on silica-based PLC," Electron. Lett., vol. 32, no. 9, pp. 847-848, 1996.

[2] G. Nakagawa, T. Yamamoto, S. Sasaki, M. Norimatsu, N. Yamamoto, T. Nosaka, K. Terada, K. Tanaka, K. Miura, and M. Yano, "High power and high sensitivity planar lightwave circuit module incorporating a novel passive alignment method," J. Lightw. Technol., vol. 16, no. 1, pp. 66-72, Jan. 1998.

[3] T. Hashimoto, T. Kurosaki, M. Yanagisawa, Y. Suzuki, Y. Akahori, Y. Inoue, Y. Tohmori, K. Kato, Y. Yamada, N. Ishihara, and K. Kato, "A $1.3 / 1.55-\mu \mathrm{m}$ wavelength-division multiplexing optical module using a planar lightwave circuit for full duplex operation," J. Lightw. Technol., vol. 18 , no. 11 , pp. 1541-1547, Nov. 2000.

[4] M. Ukechi, T. Miyashita, A. Takahashi, R. Kaku, Y. Komine, T. Mase, T. Nishimura, S. Hirayama, K. Kameda, and K. Ito, "Polyimide waveguides equipped with dielectric multilayer filter," IEICE Trans. Electron., vol. E83-C, no. 9, pp. 1458-1459, 2000.

[5] J. Yamauchi, H. Ito, H. Nakano, M. Masuda, and S. Kawaguchi, "FDTD simulation of a multiple-layer wavelength filter embedded in an optical waveguide," Contemporary Photon. Technol., vol. P-09, pp. 97-98, 2001.

[6] Y. Chung and N. Dagli, "Analysis of integrated optical corner reflectors using a finite-difference beam propagation method," IEEE Photon. Technol. Lett., vol. 3, no. 2, pp. 150-152, Feb. 1991.

[7] J. Yamauchi, Propagating Beam Analysis of Optical Waveguides, U.K.: Res. Studies Press, 2003.

[8] G. R. Hadley, "Wide-angle beam propagation using Padé approximant operators," Opt. Lett., vol. 17, no. 20, pp. 1426-1428, 1992.

[9] Y. P. Chiou, Y. C. Chiang, and H. C. Chang, "Improved three-point formulas considering the interface conditions in the finite-difference analysis of step-index optical devices," J. Lightw. Technol., vol. 18, no. 2, pp. 243-251, Feb. 2000.

[10] J. Yamauchi, T. Murata, and H. Nakano, "Semivectorial $H$-field analysis of rib waveguides by a modified beam-propagation method based on the generalized Douglas scheme," Opt. Lett., vol. 25, no. 24, pp. 1771-1773, 2000.

[11] F. Schmidt, "An adaptive approach to the numerical solution of Fresnel's wave equation," J. Lightw. Technol., vol. 11, no. 9, pp. 1425-1434, Sep. 1993.

[12] S. T. Chu, W. P. Huang, and S. K. Chaudhuri, "Simulation and analysis of waveguide based optical integrated circuits," Comput. Phys. Commun., vol. 68 , pp. 451-484, 1991. 